\title{
The Effect of Pore Size Distribution on Capillary Condensation in Nanoporous Media
}

Elizabeth Barsotti* and Mohammad Piri

Center of Innovation for Flow through Porous Media, Department of Petroleum Engineering, University of

Wyoming, 651 North $19^{\text {th }}$ Street, Laramie, Wyoming, USA, 82070

*Corresponding Author: ebarsott@gmail.com

25 Pages

27 Tables

0 Figures 
1. Raw data from the XPE-505C Mass Comparator regarding the amounts of each MCM-41 Sample in each core holder.

\begin{tabular}{|c|c|c|c|c|}
\hline \multicolumn{5}{|c|}{ S+M Mixture } \\
\hline Measurement \# & $\begin{array}{c}\text { Mass of Empty } \\
\text { Core Holder* }\end{array}$ & $\begin{array}{c}\text { Mass with MCM-41- } \\
\text { S [g] }\end{array}$ & $\begin{array}{c}\text { Mass with MCM-41- } \\
\text { M [g] }\end{array}$ & $\begin{array}{c}\text { Mass with MCM- } \\
\text { 41-L [g] }\end{array}$ \\
\hline 1 & 291.29003 & 296.25045 & 299.76833 & - \\
\hline 2 & 291.29004 & 296.25029 & 299.76826 & - \\
\hline 3 & 291.29051 & 296.25033 & 299.76834 & - \\
\hline Average & 291.29019 & 296.25036 & 299.76831 & - \\
\hline Absolute Amount & 0 & 4.96017 & 3.51795 & 0 \\
\hline
\end{tabular}

The mass of the empty core holder includes the end caps, spring, filters, and thread tape. Mixing order: S then M.

\begin{tabular}{|c|c|c|c|c|}
\hline \multicolumn{5}{|c|}{ S+M+L Mixture } \\
\hline Measurement \# & $\begin{array}{c}\text { Mass of Empty } \\
\text { Core Holder* } \\
{[\mathrm{g}]}\end{array}$ & $\begin{array}{c}\text { Mass with MCM-41- } \\
\mathrm{S}[\mathrm{g}]\end{array}$ & $\begin{array}{c}\text { Mass with MCM-41- } \\
\text { M [g] }\end{array}$ & $\begin{array}{c}\text { Mass with MCM- } \\
41-\mathrm{L}[\mathrm{g}]\end{array}$ \\
\hline 1 & 366.77398 & 368.57976 & 374.60975 & 371.23688 \\
\hline 2 & 366.77318 & 368.57891 & 374.60980 & 371.23657 \\
\hline 3 & 366.77334 & 368.57940 & 374.60963 & 371.23647 \\
\hline Average & 366.77350 & 368.57936 & 374.60973 & 371.23664 \\
\hline Absolute Amount & 0 & 1.80586 & 3.37309 & 2.65728 \\
\hline
\end{tabular}

*The mass of the empty core holder includes the end caps, spring, filters, and thread tape. Mixing order: S then L then $\mathrm{M}$.

\begin{tabular}{|c|c|c|c|c|}
\hline \multicolumn{5}{|c|}{ S+L Mixture } \\
\hline Measurement \# & $\begin{array}{c}\text { Mass of Empty } \\
\text { Core Holder* } \\
{[\mathrm{g}]}\end{array}$ & $\begin{array}{c}\text { Mass with MCM-41- } \\
\text { S [g] }\end{array}$ & $\begin{array}{c}\text { Mass with MCM-41- } \\
\text { M [g] }\end{array}$ & $\begin{array}{c}\text { Mass with MCM- } \\
\text { 41-L [g] }\end{array}$ \\
\hline 1 & 289.84398 & 294.86635 & - & 297.18625 \\
\hline 2 & 289.84406 & 294.86669 & - & 297.18623 \\
\hline 3 & 289.84415 & 294.86666 & - & 297.18640 \\
\hline Average & 289.84406 & 294.86657 & 0 & 297.18629 \\
\hline Absolute Amount & 0 & 5.02251 & - & 2.31972 \\
\hline
\end{tabular}

*The mass of the empty core holder includes the end caps, spring, filters, and thread tape. Mixing order: S then L.

2. Data for the propane isotherms measured in the $\mathrm{S}+\mathrm{L}$ mixture

\begin{tabular}{|l|c|c|c|}
\hline \multicolumn{3}{|c|}{$2.2{ }^{\circ} \mathrm{C}$} \\
\hline \multicolumn{2}{|c|}{ Adsorption } & \multicolumn{2}{c|}{ Desorption } \\
\hline Mass [g] & Pressure [bar] & Mass [g] & Pressure [bar] \\
\hline
\end{tabular}




\begin{tabular}{|c|c|c|c|}
\hline 0 & 0 & 4.752728 & 5.269418 \\
\hline 0.1329 & 0.101278 & 4.442254 & 5.114834 \\
\hline 0.400814 & 0.384041 & 3.955936 & 5.018579 \\
\hline 0.735259 & 0.848316 & 3.661372 & 5.000687 \\
\hline 0.852355 & 1.048941 & 3.406716 & 4.970661 \\
\hline 1.024051 & 1.330253 & 3.113805 & 4.811674 \\
\hline 1.093208 & 1.445328 & 3.033253 & 4.595547 \\
\hline 1.228071 & 1.663624 & 2.902032 & 4.318366 \\
\hline 1.355871 & 1.833088 & 2.81254 & 3.926998 \\
\hline 1.489673 & 1.957875 & 2.778988 & 3.86114 \\
\hline 1.623569 & 2.043729 & 2.72105 & 3.80988 \\
\hline 1.747022 & 2.119957 & 2.662654 & 3.756568 \\
\hline 1.877627 & 2.186086 & 2.590648 & 3.683234 \\
\hline 2.008191 & 2.301204 & 2.499483 & 3.607313 \\
\hline 2.11514 & 2.534737 & 2.450698 & 3.562848 \\
\hline 2.214558 & 2.854102 & 2.37646 & 3.443303 \\
\hline 2.313307 & 3.174428 & 2.323199 & 3.242869 \\
\hline 2.396016 & 3.458955 & 2.236018 & 2.951388 \\
\hline 2.482108 & 3.664222 & 2.185279 & 2.767728 \\
\hline 2.559259 & 3.748437 & 2.123354 & 2.53891 \\
\hline 2.646019 & 3.80754 & 2.07492 & 2.403546 \\
\hline 2.713245 & 3.889521 & 2.016541 & 2.26794 \\
\hline 2.791061 & 3.952946 & 1.999695 & 2.229435 \\
\hline 2.861335 & 4.100659 & 1.951379 & 2.164326 \\
\hline 2.911732 & 4.319695 & 1.929667 & 2.133733 \\
\hline 2.952707 & 4.529528 & 1.859915 & 2.075138 \\
\hline 3.000368 & 4.695898 & 1.822667 & 2.058265 \\
\hline 3.039062 & 4.831936 & 1.766523 & 2.025642 \\
\hline 3.079135 & 4.93728 & 1.638183 & 1.952194 \\
\hline 3.131945 & 5.044727 & 1.574924 & 1.919192 \\
\hline 3.289243 & 5.162468 & 1.50644 & 1.871594 \\
\hline 3.776717 & 5.22243 & 1.401272 & 1.800021 \\
\hline 4.199532 & 5.25843 & 1.33438 & 1.737821 \\
\hline \multirow[t]{5}{*}{4.752728} & 5.269418 & 1.251988 & 1.636618 \\
\hline & & 1.156768 & 1.505653 \\
\hline & & 1.087984 & 1.394848 \\
\hline & & 0.93518 & 1.145878 \\
\hline & & 0.847056 & 1.02412 \\
\hline
\end{tabular}

\section{$5.4^{\circ} \mathrm{C}$}




\begin{tabular}{|c|c|c|c|}
\hline \multicolumn{2}{|c|}{ Adsorption } & \multicolumn{2}{|c|}{ Desorption } \\
\hline Mass [g] & Pressure [bar] & Mass [g] & Pressure [bar] \\
\hline 0 & 0 & 4.398255 & 5.739994 \\
\hline 0.267457 & 0.220348 & 4.293114 & 5.713944 \\
\hline 0.558719 & 0.601786 & 4.129746 & 5.688315 \\
\hline 0.791761 & 0.989607 & 3.950746 & 5.636473 \\
\hline 0.9302 & 1.229366 & 3.872871 & 5.614451 \\
\hline 1.007011 & 1.385827 & 3.647701 & 5.565999 \\
\hline 1.203542 & 1.743764 & 3.474139 & 5.526036 \\
\hline 1.34225 & 1.93903 & 3.25522 & 5.459711 \\
\hline 1.444422 & 2.059718 & 3.102662 & 5.252729 \\
\hline 1.579777 & 2.182647 & 3.066325 & 5.147957 \\
\hline 1.82065 & 2.338011 & 2.970547 & 4.741704 \\
\hline 2.005093 & 2.514326 & 2.867651 & 4.309425 \\
\hline 2.073737 & 2.644012 & 2.826868 & 4.145383 \\
\hline 2.338801 & 3.549498 & 2.755012 & 4.056508 \\
\hline 2.395597 & 3.754874 & 2.738475 & 4.041674 \\
\hline 2.452212 & 3.921484 & 2.708075 & 4.023862 \\
\hline 2.505926 & 4.031853 & 2.633076 & 4.007671 \\
\hline 2.581896 & 4.106248 & 2.558077 & 3.929826 \\
\hline 2.80163 & 4.310148 & 2.509045 & 3.897962 \\
\hline 2.874089 & 4.479554 & 2.469084 & 3.854689 \\
\hline 2.951882 & 4.886057 & 2.413713 & 3.767841 \\
\hline 3.031931 & 5.181736 & 2.369957 & 3.601113 \\
\hline 3.126666 & 5.480981 & 2.306917 & 3.39046 \\
\hline 3.238548 & 5.596741 & 2.180012 & 2.914413 \\
\hline 3.39594 & 5.651569 & 2.134373 & 2.74845 \\
\hline 3.626371 & 5.678349 & 2.106231 & 2.653571 \\
\hline 4.064252 & 5.71614 & 2.064746 & 2.552479 \\
\hline 4.398255 & 5.739994 & 2.005603 & 2.458643 \\
\hline & & 1.957147 & 2.376772 \\
\hline & & 1.860758 & 2.302834 \\
\hline & & 1.812226 & 2.267618 \\
\hline & & 1.697565 & 2.190124 \\
\hline & & 1.629936 & 2.14684 \\
\hline & & 1.536414 & 2.089373 \\
\hline & & 1.440514 & 2.005369 \\
\hline & & 1.354244 & 1.91832 \\
\hline & & 1.238334 & 1.761354 \\
\hline & & 1.122489 & 1.579958 \\
\hline & & 0.941076 & 1.238166 \\
\hline
\end{tabular}




\begin{tabular}{|c|c|c|c|}
\hline \multicolumn{4}{|c|}{$10.0^{\circ} \mathrm{C}$} \\
\hline \multicolumn{2}{|c|}{ Adsorption } & \multicolumn{2}{|c|}{ Desorption } \\
\hline Mass [g] & Pressure [bar] & Mass [g] & Pressure [bar] \\
\hline 0 & 0 & 5.44838 & 6.636682 \\
\hline 0.321779 & 0.34034 & 5.275502 & 6.580402 \\
\hline 0.525863 & 0.642402 & 4.962861 & 6.468269 \\
\hline 0.669782 & 0.894087 & 4.231694 & 6.330272 \\
\hline 0.772564 & 1.1083 & 3.679525 & 6.303807 \\
\hline 0.930798 & 1.418735 & 3.487011 & 6.261459 \\
\hline 1.062293 & 1.677281 & 3.10265 & 5.798666 \\
\hline 1.19004 & 1.938339 & 3.040529 & 5.527006 \\
\hline 1.306799 & 2.167737 & 2.960575 & 5.146655 \\
\hline 1.42919 & 2.342949 & 2.883091 & 4.825426 \\
\hline 1.540371 & 2.469188 & 2.835715 & 4.778252 \\
\hline 1.679767 & 2.59715 & 2.796845 & 4.719623 \\
\hline 1.747405 & 2.660124 & 2.663009 & 4.628071 \\
\hline 1.832722 & 2.718834 & 2.603824 & 4.557005 \\
\hline 1.928376 & 2.787933 & 2.570577 & 4.533438 \\
\hline 2.02169 & 2.914692 & 2.487295 & 4.461785 \\
\hline 2.067328 & 3.008195 & 2.447518 & 4.335885 \\
\hline 2.158908 & 3.277181 & 2.400204 & 4.177718 \\
\hline 2.231839 & 3.538301 & 2.341053 & 3.91677 \\
\hline 2.313803 & 3.850567 & 2.268679 & 3.634197 \\
\hline 2.384812 & 4.120851 & 2.154547 & 3.246402 \\
\hline 2.446827 & 4.356236 & 2.078353 & 2.993888 \\
\hline 2.496988 & 4.578317 & 2.04506 & 2.919237 \\
\hline 2.566238 & 4.693077 & 1.9111 & 2.728392 \\
\hline 2.636676 & 4.755212 & 1.854637 & 2.673429 \\
\hline 2.680628 & 4.797285 & 1.774988 & 2.614807 \\
\hline 2.747297 & 4.89714 & 1.688601 & 2.546373 \\
\hline 2.799415 & 4.986711 & 1.567804 & 2.44874 \\
\hline 2.856371 & 5.038476 & 1.474486 & 2.357576 \\
\hline 2.933341 & 5.297102 & 1.386598 & 2.251828 \\
\hline 2.959128 & 5.449295 & 1.284909 & 2.10432 \\
\hline 3.014935 & 5.680889 & 1.167937 & 1.875526 \\
\hline 3.073334 & 5.944397 & 0.945004 & 1.427964 \\
\hline 3.13059 & 6.155144 & 0.795277 & 1.136942 \\
\hline 3.166171 & 6.279279 & & \\
\hline 3.329399 & 6.457063 & & \\
\hline 3.617336 & 6.516667 & & \\
\hline 3.941977 & 6.555251 & & \\
\hline
\end{tabular}




\begin{tabular}{|c|c|l|l|}
\hline 4.482906 & 6.593788 & & \\
\hline 5.44838 & 6.636682 & & \\
\hline
\end{tabular}

\begin{tabular}{|c|c|c|c|}
\hline \multicolumn{4}{|c|}{$\mathbf{1 6 . 0}{ }^{\circ} \mathbf{C}$} \\
\hline \multicolumn{2}{|c|}{ Adsorption } & \multicolumn{2}{c|}{ Desorption } \\
\hline Mass [g] & Pressure [bar] & Mass [g] & Pressure [bar] \\
\hline 0 & 0 & 6.049544 & 7.877068 \\
\hline 0.271546 & 0.320563 & 5.785392 & 7.806304 \\
\hline 0.560307 & 0.849605 & 5.685386 & 7.728204 \\
\hline 0.729118 & 1.196977 & 5.315482 & 7.647881 \\
\hline 0.85087 & 1.485527 & 4.842196 & 7.540152 \\
\hline 0.995725 & 1.825744 & 3.989857 & 7.42978 \\
\hline 1.111165 & 2.133077 & 3.674004 & 7.408574 \\
\hline 1.241173 & 2.42819 & 3.394734 & 7.334456 \\
\hline 1.372047 & 2.701514 & 3.130446 & 6.782174 \\
\hline 1.459962 & 2.87425 & 3.093429 & 6.570246 \\
\hline 1.625583 & 3.10258 & 3.034602 & 6.332475 \\
\hline 1.712624 & 3.201726 & 2.997517 & 6.145557 \\
\hline 1.80227 & 3.300352 & 2.945247 & 5.899996 \\
\hline 1.88103 & 3.398747 & 2.911958 & 5.850324 \\
\hline 1.964438 & 3.503104 & 2.851143 & 5.686114 \\
\hline 2.04103 & 3.625864 & 2.831638 & 5.652889 \\
\hline 2.094308 & 3.750151 & 2.732976 & 5.594972 \\
\hline 2.183586 & 3.992915 & 2.654865 & 5.517325 \\
\hline 2.248082 & 4.275422 & 2.525619 & 5.394105 \\
\hline 2.312252 & 4.56131 & 2.478208 & 5.2334 \\
\hline 2.375021 & 4.865704 & 2.399234 & 4.914152 \\
\hline 2.468793 & 5.251829 & 2.327111 & 4.592198 \\
\hline 2.534626 & 5.493226 & 2.252383 & 4.277365 \\
\hline 2.594453 & 5.657978 & 2.206479 & 4.072804 \\
\hline 2.640885 & 5.741608 & 2.125569 & 3.750843 \\
\hline 2.703089 & 5.825076 & 2.073302 & 3.623006 \\
\hline 2.776831 & 5.904818 & 2.029223 & 3.524433 \\
\hline 2.82014 & 5.976617 & 1.990777 & 3.445917 \\
\hline 2.871594 & 6.045633 & 1.891906 & 3.313163 \\
\hline 2.925823 & 6.147304 & 1.819921 & 3.235184 \\
\hline 2.963025 & 6.336557 & 1.707444 & 3.111864 \\
\hline 3.022975 & 6.599126 & 1.593833 & 2.992105 \\
\hline 3.16362 & 7.306961 & 1.506438 & 2.891016 \\
\hline 3.214517 & 7.452308 & 1.392976 & 2.721096 \\
\hline
\end{tabular}




\begin{tabular}{|l|c|c|c|}
3.402383 & 7.674401 & 1.310587 & 2.562019 \\
\hline 3.696045 & 7.74512 & 1.198213 & 2.318517 \\
\hline 4.133406 & 7.798229 & 0.927754 & 1.696956 \\
\hline 4.802721 & 7.850136 & & \\
\hline 6.049544 & 7.877068 & & \\
\hline
\end{tabular}

3. Data for the propane isotherms measured in the $\mathrm{S}+\mathrm{M}+\mathrm{L}$ mixture

\begin{tabular}{|c|c|c|c|}
\hline \multicolumn{4}{|c|}{$\mathbf{2 . 2}{ }^{\circ} \mathbf{C}$} \\
\hline \multicolumn{2}{|c|}{ Adsorption } & \multicolumn{2}{c|}{ Desorption } \\
\hline Mass [g] & Pressure [bar] & Mass [g] & Pressure [bar] \\
\hline 0 & 0 & 4.47976 & 5.206061 \\
\hline 0.245703 & 0.165312 & 4.240432 & 5.128206 \\
\hline 0.475717 & 0.42067 & 4.006068 & 5.058527 \\
\hline 0.613802 & 0.592309 & 3.590741 & 4.956321 \\
\hline 0.787267 & 0.866957 & 3.38606 & 4.758004 \\
\hline 0.855149 & 0.967977 & 3.199404 & 4.180462 \\
\hline 1.005167 & 1.210888 & 3.166601 & 4.071103 \\
\hline 1.086058 & 1.352542 & 3.093171 & 3.97426 \\
\hline 1.171388 & 1.494171 & 3.0665 & 3.943144 \\
\hline 1.279782 & 1.665256 & 2.985505 & 3.71873 \\
\hline 1.412416 & 1.834042 & 2.955835 & 3.799111 \\
\hline 1.487299 & 1.921422 & 2.894674 & 3.654248 \\
\hline 1.584031 & 2.011825 & 2.803795 & 3.606089 \\
\hline 1.659793 & 2.074997 & 2.760735 & 3.646026 \\
\hline 1.757381 & 2.147247 & 2.700105 & 3.531045 \\
\hline 1.87635 & 2.198732 & 2.687017 & 3.558151 \\
\hline 1.976264 & 2.241773 & 2.614543 & 3.466479 \\
\hline 2.059371 & 2.285631 & 2.601835 & 3.483652 \\
\hline 2.160952 & 2.39692 & 2.532596 & 3.34644 \\
\hline 2.220659 & 2.514986 & 2.524324 & 3.316223 \\
\hline 2.303336 & 2.709795 & 2.460692 & 3.122064 \\
\hline 2.387232 & 2.978898 & 2.44977 & 3.100486 \\
\hline 2.456796 & 3.190592 & 2.375973 & 2.884253 \\
\hline 2.519182 & 3.377084 & 2.318548 & 2.687256 \\
\hline 2.593088 & 3.561884 & 2.196917 & 2.412552 \\
\hline 2.654257 & 3.632109 & 2.146137 & 2.31102 \\
\hline 2.716793 & 3.683315 & 2.097593 & 2.240659 \\
\hline 2.773229 & 3.723357 & 2.033155 & 2.20076 \\
\hline 2.827705 & 3.766818 & 1.979667 & 2.18053 \\
\hline
\end{tabular}




\begin{tabular}{|c|c|c|c|}
2.883818 & 3.823288 & 1.931521 & 2.163278 \\
\hline 2.948219 & 3.868374 & 1.833098 & 2.121801 \\
\hline 3.345717 & 4.902278 & 1.796379 & 2.104369 \\
\hline 3.006792 & 3.912048 & 1.703321 & 2.049964 \\
\hline 3.052702 & 4.066993 & 1.655885 & 2.012578 \\
\hline 3.102985 & 4.241681 & 1.60666 & 1.973384 \\
\hline 3.147661 & 4.40841 & 1.519377 & 1.900628 \\
\hline 3.192775 & 4.590534 & 1.433546 & 1.815144 \\
\hline 3.235594 & 4.717318 & 1.356344 & 1.73023 \\
\hline 3.310557 & 4.895734 & 1.269825 & 1.595301 \\
\hline 3.636118 & 5.130629 & 1.175341 & 1.46817 \\
\hline 4.050361 & 5.188944 & 1.093987 & 1.339905 \\
\hline 4.47976 & 5.206061 & 0.931309 & 1.069974 \\
\hline
\end{tabular}

\begin{tabular}{|c|c|c|c|}
\hline \multicolumn{5}{|c|}{$\mathbf{5 . 4}^{\circ} \mathbf{C}$} \\
\hline \multicolumn{2}{|c|}{ Adsorption } & \multicolumn{2}{c|}{ Desorption } \\
\hline Mass [g] & Pressure [bar] & Mass [g] & Pressure [bar] \\
\hline 0 & 0 & 4.735297 & 5.725682 \\
\hline 0.419481 & 0.398295 & 4.481209 & 5.643848 \\
\hline 0.548072 & 0.581542 & 4.301238 & 5.596824 \\
\hline 0.759663 & 0.92405 & 3.981727 & 5.527174 \\
\hline 0.866239 & 1.117231 & 3.695381 & 5.470369 \\
\hline 1.036903 & 1.407757 & 3.55543 & 5.413837 \\
\hline 1.137924 & 1.602462 & 3.382781 & 5.19991 \\
\hline 1.238313 & 1.782764 & 3.29263 & 4.899086 \\
\hline 1.340878 & 1.964761 & 3.179972 & 4.473604 \\
\hline 1.44361 & 2.102313 & 3.077698 & 4.134032 \\
\hline 1.599398 & 2.266479 & 2.971841 & 4.083509 \\
\hline 1.753536 & 2.391141 & 2.881846 & 4.008545 \\
\hline 1.843705 & 2.454878 & 2.780946 & 3.962612 \\
\hline 2.004518 & 2.530135 & 2.688041 & 3.886392 \\
\hline 2.096958 & 2.617244 & 2.584034 & 3.819776 \\
\hline 2.114996 & 2.649239 & 2.510073 & 3.590087 \\
\hline 2.248433 & 2.910718 & 2.430541 & 3.336566 \\
\hline 2.298919 & 3.028286 & 2.350979 & 3.107847 \\
\hline 2.424815 & 3.445633 & 2.2782 & 2.893893 \\
\hline 2.545346 & 3.828064 & 2.216214 & 2.718059 \\
\hline 2.622302 & 4.010107 & 2.155277 & 2.605887 \\
\hline 2.697942 & 4.095881 & 2.098694 & 2.513323 \\
\hline 2.810641 & 4.181553 & 2.037311 & 2.45818 \\
\hline
\end{tabular}




\begin{tabular}{|c|c|c|c|}
2.900351 & 4.278589 & 1.982477 & 2.434348 \\
\hline 3.003022 & 4.364416 & 1.842683 & 2.37207 \\
\hline 3.066389 & 4.546026 & 1.787111 & 2.345285 \\
\hline 3.126567 & 4.783469 & 1.737372 & 2.311981 \\
\hline 3.189457 & 5.024914 & 1.615128 & 2.223414 \\
\hline 3.300462 & 5.351306 & 1.493618 & 2.109036 \\
\hline 3.473641 & 5.606847 & 1.397646 & 1.992306 \\
\hline 3.583642 & 5.639022 & 1.342282 & 1.936996 \\
\hline 3.815186 & 5.733948 & 1.20564 & 1.710908 \\
\hline 4.303676 & 5.7652 & 1.024387 & 1.374759 \\
\hline 4.841076 & 5.783996 & & \\
\hline 4.735297 & 5.738605 & & \\
\hline
\end{tabular}

\begin{tabular}{|c|c|c|c|}
\hline \multicolumn{4}{|c|}{$\mathbf{1 0 . 0}{ }^{\circ} \mathbf{C}$} \\
\hline \multicolumn{2}{|c|}{ Adsorption } & \multicolumn{2}{c|}{ Desorption } \\
\hline Mass [g] & Pressure [bar] & Mass [g] & Pressure [bar] \\
\hline 0 & 0 & 5.550817 & 6.592797 \\
\hline 0.250916 & 0.2256 & 5.323342 & 6.510636 \\
\hline 0.47074 & 0.551956 & 4.907792 & 6.397954 \\
\hline 0.655085 & 0.869821 & 4.305956 & 6.347004 \\
\hline 0.791869 & 1.128561 & 4.161311 & 6.318714 \\
\hline 0.967803 & 1.476594 & 3.831447 & 6.262245 \\
\hline 1.067253 & 1.691993 & 3.71776 & 6.230839 \\
\hline 1.2013 & 1.963253 & 3.549908 & 6.15781 \\
\hline 1.360186 & 2.262154 & 3.368424 & 5.879389 \\
\hline 1.521304 & 2.491555 & 3.244675 & 5.403049 \\
\hline 1.633838 & 2.646226 & 3.119067 & 4.919853 \\
\hline 1.744257 & 2.742555 & 3.012066 & 4.759807 \\
\hline 1.936925 & 2.883426 & 2.905944 & 4.668119 \\
\hline 2.07758 & 2.973562 & 2.799041 & 4.58115 \\
\hline 2.187933 & 3.164909 & 2.668675 & 4.496177 \\
\hline 2.269541 & 3.359696 & 2.560319 & 4.315702 \\
\hline 2.36511 & 3.660675 & 2.470666 & 4.004656 \\
\hline 2.495155 & 4.168829 & 2.396204 & 3.702056 \\
\hline 2.606493 & 4.498662 & 2.365973 & 3.424163 \\
\hline 2.640358 & 4.569731 & 2.290241 & 3.208143 \\
\hline 2.701454 & 4.655569 & 2.210793 & 3.052852 \\
\hline 2.742665 & 4.682588 & 2.133344 & 2.936012 \\
\hline 2.800284 & 4.736297 & 2.072651 & 2.872083 \\
\hline 2.839258 & 4.788991 & 1.992011 & 2.82996 \\
\hline
\end{tabular}




\begin{tabular}{|c|c|c|c|}
2.895585 & 4.88068 & 1.884337 & 2.768646 \\
\hline 2.972502 & 4.943488 & 1.775815 & 2.68791 \\
\hline 3.085435 & 5.16626 & 1.675344 & 2.589058 \\
\hline 3.127479 & 5.370445 & 1.579893 & 2.472355 \\
\hline 3.175902 & 5.560597 & 1.481124 & 2.362759 \\
\hline 3.222729 & 5.757323 & 1.318579 & 2.101345 \\
\hline 3.291954 & 5.986866 & 1.186761 & 1.834103 \\
\hline 3.39559 & 6.242574 & 0.951182 & 1.392647 \\
\hline 3.547441 & 6.405519 & 0.818135 & 1.126526 \\
\hline 3.906307 & 6.504929 & & \\
\hline 4.290087 & 6.554854 & & \\
\hline 4.832335 & 6.577589 & & \\
\hline 5.550817 & 6.592797 & & \\
\hline
\end{tabular}

\begin{tabular}{|c|c|c|c|}
\hline \multicolumn{4}{|c|}{$\mathbf{1 6 . 0}{ }^{\circ} \mathbf{C}$} \\
\hline \multicolumn{2}{|c|}{ Adsorption } & \multicolumn{2}{c|}{ Desorption } \\
\hline Mass [g] & Pressure [bar] & Mass [g] & Pressure [bar] \\
\hline 0 & 0 & 5.613746 & 7.826988 \\
\hline 0.288666 & 0.353089 & 5.38653 & 7.726346 \\
\hline 0.572374 & 0.865518 & 4.94275 & 7.588197 \\
\hline 0.728095 & 1.195828 & 4.461277 & 7.529523 \\
\hline 0.877091 & 1.549275 & 4.264939 & 7.49552 \\
\hline 1.021074 & 1.900974 & 3.89893 & 7.434968 \\
\hline 1.158166 & 2.239272 & 3.728453 & 7.39218 \\
\hline 1.306367 & 2.567001 & 3.564943 & 7.280292 \\
\hline 1.429966 & 2.832613 & 3.385182 & 6.842078 \\
\hline 1.566296 & 3.06886 & 3.240493 & 6.214748 \\
\hline 1.692105 & 3.258755 & 3.115613 & 5.822463 \\
\hline 1.808735 & 3.413368 & 2.975396 & 5.641196 \\
\hline 1.968037 & 3.557842 & 2.838687 & 5.527397 \\
\hline 2.088801 & 3.664674 & 2.703094 & 5.416629 \\
\hline 2.20923 & 3.837313 & 2.582459 & 5.084475 \\
\hline 2.319151 & 4.129694 & 2.464462 & 4.682107 \\
\hline 2.417846 & 4.470381 & 2.370431 & 4.310056 \\
\hline 2.505323 & 4.820839 & 2.277766 & 3.995242 \\
\hline 2.670419 & 5.439885 & 2.195005 & 3.794618 \\
\hline 2.759212 & 5.627395 & 2.104916 & 3.616326 \\
\hline 2.837825 & 5.715365 & 2.02472 & 3.524824 \\
\hline 2.908418 & 5.795082 & 1.948559 & 3.466036 \\
\hline 2.989948 & 5.900417 & 1.863518 & 3.398099 \\
\hline
\end{tabular}




\begin{tabular}{|c|c|c|c|}
3.046732 & 5.957182 & 1.788251 & 3.322158 \\
\hline 3.124305 & 6.125949 & 1.714026 & 3.232799 \\
\hline 3.14996 & 6.275545 & 1.638696 & 3.144964 \\
\hline 3.200762 & 6.520212 & 1.567135 & 3.041536 \\
\hline 3.279226 & 6.85107 & 1.455127 & 2.867019 \\
\hline 3.409943 & 7.327374 & 1.351456 & 2.66961 \\
\hline 3.468381 & 7.465554 & 1.177364 & 2.293503 \\
\hline 3.649195 & 7.630494 & 0.894164 & 1.647015 \\
\hline 3.888015 & 7.715218 & & \\
\hline 4.387215 & 7.750985 & & \\
\hline 4.963884 & 7.79898 & & \\
\hline 5.613746 & 7.826988 & & \\
\hline
\end{tabular}

4. Data for the propane isotherms measured in the $\mathrm{S}+\mathrm{M}$ mixture

\begin{tabular}{|c|c|c|c|}
\hline \multicolumn{4}{|c|}{$\mathbf{2 . 2}^{\circ} \mathbf{C}$} \\
\hline \multicolumn{2}{|c|}{ Adsorption } & \multicolumn{2}{c|}{ Desorption } \\
\hline Mass [g] & Pressure [bar] & Mass [g] & Pressure [bar] \\
\hline 0 & 0 & 5.130292 & 5.33008 \\
\hline 0.26797 & 0.165456 & 5.00093 & 5.283037 \\
\hline 0.537268 & 0.423011 & 4.354413 & 5.081244 \\
\hline 0.691433 & 0.592876 & 4.038915 & 5.032025 \\
\hline 0.913232 & 0.869843 & 3.768035 & 4.965576 \\
\hline 1.03358 & 0.955192 & 3.575899 & 4.756519 \\
\hline 1.178631 & 1.15569 & 3.330946 & 4.260787 \\
\hline 1.269963 & 1.273693 & 3.20087 & 3.809564 \\
\hline 1.351943 & 1.394208 & 3.139234 & 3.711122 \\
\hline 1.46995 & 1.539811 & 3.03167 & 3.20918 \\
\hline 1.59897 & 1.703235 & 2.981466 & 2.971444 \\
\hline 1.69885 & 1.808119 & 2.935077 & 2.777248 \\
\hline 1.800836 & 1.896928 & 2.887043 & 2.618923 \\
\hline 1.910892 & 1.963169 & 2.849452 & 2.499791 \\
\hline 2.010676 & 2.028589 & 2.807582 & 2.41655 \\
\hline 2.135427 & 2.085147 & 2.767987 & 2.338717 \\
\hline 2.224174 & 2.144173 & 2.727499 & 2.28712 \\
\hline 2.347676 & 2.199424 & 2.682199 & 2.242998 \\
\hline 2.465338 & 2.258003 & 2.596306 & 2.198324 \\
\hline 2.585878 & 2.309804 & 2.553744 & 2.187694 \\
\hline 2.706053 & 2.356895 & 2.499795 & 2.152703 \\
\hline 2.814218 & 2.436029 & 2.392097 & 2.112987 \\
\hline
\end{tabular}




\begin{tabular}{|c|c|c|c|}
\hline 2.900975 & 2.558807 & 2.312475 & 2.078775 \\
\hline 2.976157 & 2.729473 & 2.181315 & 2.018021 \\
\hline 3.045135 & 2.964855 & 2.078283 & 1.970181 \\
\hline 3.111152 & 3.228738 & 1.95688 & 1.913974 \\
\hline 3.176462 & 3.543875 & 1.838433 & 1.848341 \\
\hline 3.225249 & 3.82962 & 1.720427 & 1.769682 \\
\hline 3.276054 & 4.079737 & 1.61079 & 1.673008 \\
\hline 3.322076 & 4.296451 & 1.50748 & 1.581423 \\
\hline 3.357385 & 4.48814 & 1.423924 & 1.464155 \\
\hline 3.415316 & 4.745217 & 1.332715 & 1.352759 \\
\hline 3.502016 & 5.007006 & 1.239177 & 1.233585 \\
\hline 3.58745 & 4.969713 & 1.149878 & 1.116091 \\
\hline 3.659326 & 5.200499 & 1.05985 & 0.999305 \\
\hline 4.081129 & 5.288756 & 1.076562 & 1.016261 \\
\hline 4.461682 & 5.301714 & & \\
\hline 5.130292 & 5.33008 & & \\
\hline
\end{tabular}

\begin{tabular}{|c|c|c|c|}
\hline \multicolumn{5}{|c|}{$\mathbf{5 . 4}{ }^{\circ} \mathbf{C}$} \\
\hline \multicolumn{2}{|c|}{ Adsorption } & \multicolumn{2}{c|}{ Desorption } \\
\hline Mass [g] & Pressure [bar] & Mass [g] & Pressure [bar] \\
\hline 0 & 0 & 5.669333 & 5.875957 \\
\hline 0.519336 & 0.392335 & 5.5294 & 5.809244 \\
\hline 0.672021 & 0.57325 & 5.228975 & 5.689445 \\
\hline 0.91609 & 0.866272 & 5.047718 & 5.64126 \\
\hline 1.027211 & 1.028489 & 4.571003 & 5.55752 \\
\hline 1.18904 & 1.260909 & 4.337609 & 5.539457 \\
\hline 1.349245 & 1.511227 & 4.084726 & 5.504235 \\
\hline 1.461729 & 1.661481 & 3.794574 & 5.444085 \\
\hline 1.614479 & 1.874275 & 3.593985 & 5.210959 \\
\hline 1.711689 & 1.989402 & 3.421279 & 4.625341 \\
\hline 1.874939 & 2.122882 & 3.305755 & 4.086131 \\
\hline 2.03006 & 2.228843 & 3.121057 & 3.716156 \\
\hline 2.140946 & 2.289439 & 2.929949 & 3.013272 \\
\hline 2.301035 & 2.393556 & 2.883596 & 2.871567 \\
\hline 2.402291 & 2.465887 & 2.836301 & 2.751762 \\
\hline 2.428676 & 2.48309 & 2.823911 & 2.66104 \\
\hline 2.528708 & 2.535128 & 2.776162 & 2.583371 \\
\hline 2.639289 & 2.581512 & 2.737005 & 2.524739 \\
\hline 2.801872 & 2.666405 & 2.746887 & 2.493605 \\
\hline 2.895426 & 2.804735 & 2.654902 & 2.435698 \\
\hline
\end{tabular}




\begin{tabular}{|c|c|c|c|}
2.986146 & 2.998189 & 2.563684 & 2.402386 \\
\hline 3.068526 & 3.30786 & 2.433567 & 2.354579 \\
\hline 3.138233 & 3.632579 & 2.348644 & 2.316313 \\
\hline 3.203307 & 3.972284 & 2.232342 & 2.247953 \\
\hline 3.263419 & 4.286831 & 2.113954 & 2.193187 \\
\hline 3.34882 & 4.711529 & 1.998295 & 2.135281 \\
\hline 3.42075 & 5.102388 & 1.922057 & 2.088163 \\
\hline 3.499862 & 5.389795 & 1.815213 & 2.016708 \\
\hline 3.543607 & 5.497752 & 1.695662 & 1.920433 \\
\hline 3.594669 & 5.578492 & 1.60158 & 1.811536 \\
\hline 3.741464 & 5.682714 & 1.504466 & 1.707091 \\
\hline 3.862056 & 5.723566 & 1.457796 & 1.65922 \\
\hline 4.296345 & 5.816554 & 1.286841 & 1.403512 \\
\hline 5.166239 & 5.862744 & 1.133841 & 1.178273 \\
\hline 5.669333 & 5.875957 & 0.013283 & -0.00264 \\
\hline
\end{tabular}

\begin{tabular}{|c|c|c|c|}
\hline \multicolumn{4}{|c|}{$\mathbf{1 0 . 0}{ }^{\circ} \mathbf{C}$} \\
\hline Adsorption & \multicolumn{2}{c|}{ Desorption } \\
\hline Mass [g] & Pressure [bar] & Mass [g] & Pressure [bar] \\
\hline 0 & 0 & 5.798219 & 6.607312 \\
\hline 0.337219 & 0.23137 & 5.704091 & 6.578166 \\
\hline 0.822482 & 0.852765 & 5.410538 & 6.481342 \\
\hline 0.940902 & 1.044359 & 4.989865 & 6.393057 \\
\hline 1.137075 & 1.366801 & 4.715582 & 6.356548 \\
\hline 1.301564 & 1.64274 & 4.474592 & 6.332225 \\
\hline 1.450206 & 1.889548 & 4.220923 & 6.309171 \\
\hline 1.517281 & 2.003175 & 3.981026 & 6.285236 \\
\hline 1.597392 & 2.120892 & 3.587495 & 6.078621 \\
\hline 1.773208 & 2.346425 & 3.480736 & 5.783238 \\
\hline 1.80046 & 2.369317 & 3.393653 & 5.410879 \\
\hline 1.934341 & 2.491774 & 3.292525 & 5.041142 \\
\hline 2.037894 & 2.557758 & 3.141458 & 4.470423 \\
\hline 2.150901 & 2.642277 & 3.001968 & 3.741667 \\
\hline 2.226669 & 2.684161 & 2.942754 & 3.490511 \\
\hline 2.33909 & 2.780521 & 2.889087 & 3.292238 \\
\hline 2.372076 & 2.777246 & 2.834671 & 3.150495 \\
\hline 2.465604 & 2.840973 & 2.781684 & 3.040727 \\
\hline 2.555918 & 2.896153 & 2.675035 & 2.928478 \\
\hline 2.650091 & 2.94241 & 2.59029 & 2.859369 \\
\hline 2.743096 & 3.005865 & 2.451636 & 2.789152 \\
\hline
\end{tabular}




\begin{tabular}{|l|l|l|l|}
2.835463 & 3.119504 & 2.286113 & 2.686734 \\
\hline 2.836241 & 3.089342 & 2.152238 & 2.613339 \\
\hline 2.928959 & 3.282781 & 2.030198 & 2.531759 \\
\hline 2.952269 & 3.407491 & 1.96555 & 2.457251 \\
\hline 3.013213 & 3.523979 & 1.854234 & 2.360969 \\
\hline 3.096662 & 3.909611 & 1.741763 & 2.264498 \\
\hline 3.241457 & 4.613953 & 1.642851 & 2.134722 \\
\hline 3.331846 & 5.107543 & 1.544866 & 2.019978 \\
\hline 3.381562 & 5.361669 & 1.441158 & 1.855154 \\
\hline 3.449077 & 5.707961 & 1.301075 & 1.629403 \\
\hline 3.496516 & 5.893823 & 1.065354 & 1.226788 \\
\hline 3.552955 & 6.104747 & 1.054887 & 1.239723 \\
\hline 3.598987 & 6.261293 & & \\
\hline 3.69036 & 6.417351 & & \\
\hline 3.783852 & 6.476807 & & \\
\hline 4.019565 & 6.538408 & & \\
\hline 5.558182 & 6.609223 & & \\
\hline 5.798219 & 6.607312 & & \\
\hline
\end{tabular}

\begin{tabular}{|c|c|c|c|}
\hline \multicolumn{4}{|c|}{$16.0{ }^{\circ} \mathbf{C}$} \\
\hline \multicolumn{2}{|c|}{ Adsorption } & \multicolumn{2}{c|}{ Desorption } \\
\hline Mass [g] & Pressure [bar] & Mass [g] & Pressure [bar] \\
\hline 0 & 0 & 5.320905 & 7.898994 \\
\hline 0.356074 & 0.349514 & 5.328296 & 7.880438 \\
\hline 0.698561 & 0.861045 & 4.947793 & 7.73816 \\
\hline 0.856814 & 1.141466 & 4.614952 & 7.612768 \\
\hline 1.004744 & 1.440314 & 4.089872 & 7.496667 \\
\hline 1.157938 & 1.745162 & 3.845776 & 7.454717 \\
\hline 1.34422 & 2.12164 & 3.633407 & 7.352179 \\
\hline 1.522192 & 2.473943 & 3.504938 & 7.143015 \\
\hline 1.658796 & 2.6933 & 3.378735 & 6.702342 \\
\hline 1.791788 & 2.896179 & 3.208608 & 5.842179 \\
\hline 1.938305 & 3.07269 & 3.042259 & 5.335898 \\
\hline 2.055885 & 3.197552 & 2.895475 & 4.585942 \\
\hline 2.232436 & 3.357473 & 2.839091 & 4.270689 \\
\hline 2.362358 & 3.481656 & 2.772189 & 3.994389 \\
\hline 2.474523 & 3.574761 & 2.706601 & 3.806677 \\
\hline 2.60608 & 3.661832 & 2.648602 & 3.680075 \\
\hline 2.723448 & 3.758714 & 2.586985 & 3.585108 \\
\hline 2.83694 & 3.923384 & 2.533113 & 3.529003 \\
\hline
\end{tabular}




\begin{tabular}{|c|c|c|c|}
\hline 2.937146 & 4.172629 & 2.410357 & 3.435035 \\
\hline 3.028048 & 4.535379 & 2.310338 & 3.39655 \\
\hline 3.114113 & 4.959159 & 2.194553 & 3.291419 \\
\hline 3.204757 & 5.46797 & 2.079319 & 3.201283 \\
\hline 3.266728 & 5.818118 & 1.973528 & 3.126494 \\
\hline 3.340796 & 6.236296 & 1.879091 & 3.03219 \\
\hline 3.4029 & 6.611208 & 1.682206 & 2.825533 \\
\hline 3.45296 & 6.913543 & 1.586532 & 2.714621 \\
\hline 3.501538 & 7.151179 & 1.501871 & 2.578701 \\
\hline 3.521018 & 7.350386 & 1.419055 & 2.435059 \\
\hline 3.54371 & 7.459793 & 1.206158 & 1.991993 \\
\hline 3.606468 & 7.611487 & 0.907906 & 1.419556 \\
\hline 3.845624 & 7.810852 & & \\
\hline 4.238928 & 7.865137 & & \\
\hline 4.73879 & 7.873577 & & \\
\hline 5.320905 & 7.898994 & & \\
\hline
\end{tabular}

5. Data for the $\mathrm{n}$-butane isotherms measured in the $\mathrm{S}+\mathrm{L}$ mixture

\begin{tabular}{|c|c|c|c|}
\hline \multicolumn{4}{|c|}{$\mathbf{9 . 1}^{\circ} \mathbf{C}$} \\
\hline Adsorption & \multicolumn{2}{c|}{ Desorption } \\
\hline Mass [g] & Pressure [bar] & Mass [g] & Pressure [bar] \\
\hline 0 & 0 & 5.441342 & 1.503225 \\
\hline 0.226421 & 0.04483 & 5.384491 & 1.528525 \\
\hline 0.473223 & 0.10922 & 5.263261 & 1.474817 \\
\hline 0.959366 & 0.27967 & 5.064522 & 1.448009 \\
\hline 1.123099 & 0.338241 & 4.636044 & 1.431364 \\
\hline 1.186214 & 0.367153 & 4.173394 & 1.415677 \\
\hline 1.418652 & 0.409991 & 3.734575 & 1.416705 \\
\hline 1.727201 & 0.432624 & 3.533516 & 1.41148 \\
\hline 1.728011 & 0.443686 & 3.290437 & 1.406652 \\
\hline 2.004378 & 0.478847 & 3.13065 & 1.377484 \\
\hline 2.133692 & 0.558459 & 3.076027 & 1.336542 \\
\hline 2.247777 & 0.686063 & 3.009806 & 1.262344 \\
\hline 2.318273 & 0.774972 & 2.9782 & 1.199702 \\
\hline 2.397611 & 0.874684 & 2.932975 & 1.119512 \\
\hline 2.536686 & 0.924871 & 2.92039 & 1.099038 \\
\hline 2.540407 & 0.915428 & 2.83752 & 0.9685 \\
\hline 2.587345 & 0.933702 & 2.806787 & 0.935546 \\
\hline 2.674449 & 0.959607 & 2.717589 & 0.911664 \\
\hline
\end{tabular}




\begin{tabular}{|c|c|c|c|}
\hline 2.826833 & 1.012114 & 2.599759 & 0.898559 \\
\hline 3.107977 & 1.411167 & 2.537187 & 0.878407 \\
\hline 3.549901 & 1.468419 & 2.496975 & 0.86875 \\
\hline 4.047443 & 1.484681 & 2.450727 & 0.860744 \\
\hline \multirow[t]{18}{*}{5.441342} & 1.503225 & 2.394536 & 0.836572 \\
\hline & & 2.308924 & 0.792304 \\
\hline & & 2.228184 & 0.688363 \\
\hline & & 2.199457 & 0.644075 \\
\hline & & 2.142333 & 0.572591 \\
\hline & & 2.114506 & 0.524799 \\
\hline & & 2.094874 & 0.536516 \\
\hline & & 2.055372 & 0.477331 \\
\hline & & 2.039476 & 0.492257 \\
\hline & & 1.9863 & 0.459528 \\
\hline & & 1.677516 & 0.416411 \\
\hline & & 1.462266 & 0.405577 \\
\hline & & 1.424691 & 0.394929 \\
\hline & & 1.20161 & 0.360815 \\
\hline & & 1.016387 & 0.298102 \\
\hline & & 0.853242 & 0.238663 \\
\hline & & 0.757598 & 0.204394 \\
\hline & & 0.549738 & 0.12838 \\
\hline
\end{tabular}

\begin{tabular}{|c|c|c|c|}
\hline \multicolumn{4}{|c|}{$\mathbf{1 3 . 1}^{\circ} \mathbf{C}$} \\
\hline Adsorption & \multicolumn{2}{c|}{ Desorption } \\
\hline Mass [g] & Pressure [bar] & Mass [g] & Pressure [bar] \\
\hline 0 & 0 & 4.339968 & 1.764413 \\
\hline 0.743918 & 0.23901 & 4.052057 & 1.656425 \\
\hline 0.756859 & 0.235517 & 3.75836 & 1.625165 \\
\hline 0.995856 & 0.352704 & 3.374305 & 1.603918 \\
\hline 1.242069 & 0.439661 & 3.272812 & 1.603687 \\
\hline 1.289791 & 0.443527 & 3.085427 & 1.554178 \\
\hline 1.361604 & 0.463848 & 3.040434 & 1.5142 \\
\hline 1.405417 & 0.467175 & 3.006465 & 1.465482 \\
\hline 1.660566 & 0.508889 & 2.952996 & 1.370219 \\
\hline 1.811371 & 0.521157 & 2.902706 & 1.25463 \\
\hline 1.965756 & 0.558441 & 2.864061 & 1.170748 \\
\hline 2.089628 & 0.617875 & 2.829163 & 1.139022 \\
\hline
\end{tabular}




\begin{tabular}{|c|c|c|c|}
\hline 2.18009 & 0.734301 & 2.776619 & 1.07427 \\
\hline 2.256667 & 0.85202 & 2.695043 & 1.053233 \\
\hline 2.325776 & 0.925006 & 2.496545 & 1.025688 \\
\hline 2.377328 & 0.999486 & 2.450233 & 1.008636 \\
\hline 2.471608 & 1.051184 & 2.272523 & 0.880816 \\
\hline 2.607292 & 1.081699 & 2.139298 & 0.679774 \\
\hline 2.732062 & 1.131087 & 2.050798 & 0.57962 \\
\hline 2.789053 & 1.157256 & 1.9578 & 0.535396 \\
\hline 2.848162 & 1.211689 & 1.847258 & 0.512896 \\
\hline 2.903503 & 1.309941 & 1.856815 & 0.526486 \\
\hline 2.93938 & 1.405848 & 1.597431 & 0.482103 \\
\hline 3.021805 & 1.569829 & 1.390534 & 0.460597 \\
\hline 3.163371 & 1.687504 & 1.150715 & 0.408274 \\
\hline 3.547249 & 1.726687 & 1.093797 & 0.386482 \\
\hline 3.97407 & 1.738839 & 0.958476 & 0.330074 \\
\hline 4.339968 & 1.764413 & 0.817049 & 0.263866 \\
\hline & & 0.66919 & 0.20207 \\
\hline & & 0.541396 & 0.152144 \\
\hline & & 0.405034 & 0.104368 \\
\hline
\end{tabular}

\begin{tabular}{|c|c|c|c|}
\hline \multicolumn{4}{|c|}{$16 .{ }^{\circ} \mathbf{C}$} \\
\hline \multicolumn{2}{|c|}{ Adsorption } & \multicolumn{2}{c|}{ Desorption } \\
\hline Mass [g] & Pressure [bar] & Mass [g] & Pressure [bar] \\
\hline 0 & 0 & 5.332225 & 1.925433 \\
\hline 0.46145 & 0.128996 & 5.113945 & 1.875358 \\
\hline 0.746967 & 0.25916 & 4.77873 & 1.81442 \\
\hline 1.022825 & 0.396127 & 4.140591 & 1.791111 \\
\hline 1.329214 & 0.509464 & 3.600857 & 1.769943 \\
\hline 2.315757 & 1.03286 & 3.138579 & 1.725266 \\
\hline 2.355383 & 1.081152 & 2.949218 & 1.446033 \\
\hline 2.406582 & 1.151506 & 2.747652 & 1.176411 \\
\hline 2.502161 & 1.187624 & 2.598916 & 1.141848 \\
\hline 2.614453 & 1.217845 & 2.435581 & 1.097396 \\
\hline 2.74371 & 1.278353 & 2.313012 & 0.997352 \\
\hline 2.810462 & 1.305064 & 2.228827 & 0.877582 \\
\hline 2.890537 & 1.425744 & 2.153885 & 0.749545 \\
\hline 2.935936 & 1.533809 & 2.073319 & 0.658748 \\
\hline 2.98158 & 1.651252 & 1.922971 & 0.583647 \\
\hline 3.026615 & 1.73637 & 1.829604 & 0.567805 \\
\hline 3.140608 & 1.826076 & 1.53501 & 0.527909 \\
\hline
\end{tabular}




\begin{tabular}{|c|c|c|c|}
3.649913 & 1.881428 & 1.442761 & 0.524842 \\
\hline 4.541232 & 1.907421 & 1.296742 & 0.489989 \\
\hline 5.332225 & 1.925433 & 1.113066 & 0.424535 \\
\hline & & 0.888589 & 0.31923 \\
\hline & & 0.716475 & 0.235725 \\
\hline & & 0.516529 & 0.152262 \\
\hline
\end{tabular}

\begin{tabular}{|c|c|c|c|}
\hline \multicolumn{4}{|c|}{$20.0^{\circ} \mathrm{C}$} \\
\hline \multicolumn{2}{|c|}{ Adsorption } & \multicolumn{2}{|c|}{ Desorption } \\
\hline Mass [g] & Pressure [bar] & Mass [g] & Pressure [bar] \\
\hline 0 & 0 & 3.86429 & 2.292226 \\
\hline 0.44393 & 0.150003 & 3.542002 & 2.259034 \\
\hline 0.65705 & 0.253228 & 3.454892 & 2.173095 \\
\hline 0.864624 & 0.370753 & 3.181929 & 2.036257 \\
\hline 0.905685 & 0.396798 & 2.973204 & 1.747876 \\
\hline 1.17698 & 0.54002 & 2.758351 & 1.417713 \\
\hline 1.259991 & 0.571879 & 2.59803 & 1.343077 \\
\hline 1.277025 & 0.582895 & 2.439129 & 1.286261 \\
\hline 1.402858 & 0.608939 & 2.297002 & 1.123964 \\
\hline 1.507257 & 0.636063 & 2.18665 & 0.941292 \\
\hline 1.512727 & 0.636939 & 2.063707 & 0.766776 \\
\hline 1.781142 & 0.685585 & 1.78724 & 0.658038 \\
\hline 1.818491 & 0.692343 & 1.718941 & 0.649161 \\
\hline 1.901438 & 0.704425 & 1.620618 & 0.645922 \\
\hline 2.160558 & 0.920132 & 1.39499 & 0.593326 \\
\hline 2.188123 & 0.98258 & 1.293283 & 0.570615 \\
\hline 2.230371 & 1.065249 & 1.148402 & 0.52275 \\
\hline 2.274411 & 1.143775 & 1.062719 & 0.476292 \\
\hline 2.330313 & 1.242872 & 0.917133 & 0.399803 \\
\hline 2.374852 & 1.321689 & 0.788699 & 0.321928 \\
\hline 2.408804 & 1.37245 & 0.471289 & 0.161618 \\
\hline 2.559474 & 1.429953 & & \\
\hline 2.642835 & 1.471329 & & \\
\hline 2.711054 & 1.520762 & & \\
\hline 2.786423 & 1.546526 & & \\
\hline 2.857688 & 1.66229 & & \\
\hline 2.901833 & 1.772539 & & \\
\hline 2.991141 & 2.015763 & & \\
\hline 3.213182 & 2.175187 & & \\
\hline 3.487846 & 2.248221 & & \\
\hline
\end{tabular}




\begin{tabular}{|l|l|l|l|}
\hline 3.86429 & 2.292226 & & \\
\hline
\end{tabular}

6. Data for the $\mathrm{n}$-butane isotherms measured in the $\mathrm{S}+\mathrm{M}+\mathrm{L}$ mixture

\begin{tabular}{|c|c|c|c|}
\hline \multicolumn{4}{|c|}{$\mathbf{9 . 1}{ }^{\circ} \mathbf{C}$} \\
\hline \multicolumn{2}{|c|}{ Adsorption } & \multicolumn{2}{c|}{ Desorption } \\
\hline Mass [g] & Pressure [bar] & Mass [g] & Pressure [bar] \\
\hline 0 & 0 & 5.166983 & 1.508577 \\
\hline 0.171968 & 0.027266 & 5.042096 & 1.498219 \\
\hline 0.373273 & 0.071769 & 4.897843 & 1.453708 \\
\hline 0.576795 & 0.134744 & 4.646268 & 1.421355 \\
\hline 0.803157 & 0.220071 & 4.479458 & 1.418924 \\
\hline 0.958087 & 0.26853 & 4.205001 & 1.40523 \\
\hline 1.022295 & 0.305157 & 3.937794 & 1.407303 \\
\hline 1.230155 & 0.377792 & 3.714232 & 1.390423 \\
\hline 1.392994 & 0.404784 & 3.522146 & 1.366266 \\
\hline 1.446701 & 0.428406 & 3.436293 & 1.343446 \\
\hline 1.546751 & 0.431868 & 3.368735 & 1.283553 \\
\hline 1.760674 & 0.458138 & 3.316788 & 1.23024 \\
\hline 1.806803 & 0.458808 & 3.278608 & 1.167897 \\
\hline 1.905241 & 0.472798 & 3.211067 & 1.068145 \\
\hline 2.064281 & 0.482595 & 3.188508 & 1.029699 \\
\hline 2.233721 & 0.533566 & 3.136961 & 0.959908 \\
\hline 2.357567 & 0.662215 & 3.111402 & 0.937626 \\
\hline 2.57575 & 0.878919 & 3.090879 & 0.921838 \\
\hline 2.630714 & 0.888765 & 3.024818 & 0.905454 \\
\hline 2.741015 & 0.912012 & 2.932907 & 0.888586 \\
\hline 2.89479 & 0.95169 & 2.829833 & 0.868711 \\
\hline 2.978732 & 0.984274 & 2.741487 & 0.861229 \\
\hline 3.099189 & 1.10043 & 2.628212 & 0.834892 \\
\hline 3.149193 & 1.192718 & 2.480053 & 0.798986 \\
\hline 3.182874 & 1.240779 & 2.381013 & 0.692732 \\
\hline 3.240059 & 1.313665 & 2.283206 & 0.577885 \\
\hline 3.321169 & 1.378 & 2.217537 & 0.544829 \\
\hline 3.614449 & 1.463501 & 2.340429 & 0.645014 \\
\hline 4.005357 & 1.483351 & 2.26501 & 0.566056 \\
\hline 4.611064 & 1.491198 & 2.236846 & 0.542257 \\
\hline 5.166983 & 1.508577 & 2.21503 & 0.535394 \\
\hline & & 1.983752 & 0.467446 \\
\hline & & 1.702492 & 0.454538 \\
\hline
\end{tabular}




\begin{tabular}{|l|l|l|l|} 
& & 1.464542 & 0.419329 \\
\hline & & 1.370518 & 0.403657 \\
\hline & & 1.214726 & 0.363304 \\
\hline & & 1.086001 & 0.328296 \\
\hline & & 0.882892 & 0.248109 \\
\hline
\end{tabular}

\begin{tabular}{|c|c|c|c|}
\hline \multicolumn{4}{|c|}{$\mathbf{1 3 . 1}{ }^{\circ} \mathbf{C}$} \\
\hline \multicolumn{2}{|c|}{ Adsorption } & \multicolumn{2}{c|}{ Desorption } \\
\hline Mass [g] & Pressure [bar] & Mass [g] & Pressure [bar] \\
\hline 0 & 0 & 4.149697 & 1.672451 \\
\hline 0.697861 & 0.209965 & 3.744567 & 1.608964 \\
\hline 0.760299 & 0.249242 & 3.59236 & 1.58788 \\
\hline 0.770297 & 0.248319 & 3.472849 & 1.571528 \\
\hline 1.092003 & 0.394791 & 3.389268 & 1.535567 \\
\hline 1.15865 & 0.425971 & 3.263475 & 1.394782 \\
\hline 1.280353 & 0.462838 & 3.205994 & 1.30776 \\
\hline 1.426387 & 0.496444 & 3.192507 & 1.256076 \\
\hline 1.619115 & 0.539094 & 3.163551 & 1.190436 \\
\hline 1.944416 & 0.565749 & 3.136426 & 1.17393 \\
\hline 2.189539 & 0.637249 & 3.070965 & 1.094517 \\
\hline 2.351165 & 0.806833 & 2.920405 & 1.064095 \\
\hline 2.428305 & 0.901073 & 2.686366 & 1.016849 \\
\hline 2.485792 & 0.985284 & 2.473656 & 0.942559 \\
\hline 2.545025 & 1.035544 & 2.310819 & 0.750555 \\
\hline 2.684617 & 1.060593 & 2.234611 & 0.669981 \\
\hline 2.803909 & 1.09274 & 2.132796 & 0.612753 \\
\hline 2.851577 & 1.119172 & 1.916835 & 0.557942 \\
\hline 2.949527 & 1.15274 & 1.757783 & 0.544172 \\
\hline 3.024464 & 1.194376 & 1.553992 & 0.518832 \\
\hline 3.120946 & 1.350551 & 1.376756 & 0.48043 \\
\hline 3.157501 & 1.424203 & 1.251015 & 0.459942 \\
\hline 3.20383 & 1.531614 & 1.109726 & 0.402978 \\
\hline 3.328004 & 1.632108 & 0.943031 & 0.328272 \\
\hline 3.577333 & 1.712326 & 0.751775 & 0.236965 \\
\hline 3.58645 & 1.713679 & 0.514939 & 0.134742 \\
\hline 4.005026 & 1.746622 & & \\
\hline 4.311834 & 1.753129 & & \\
\hline 4.149697 & 1.672451 & & \\
\hline
\end{tabular}




\begin{tabular}{|c|c|c|c|}
\hline \multicolumn{4}{|c|}{$16.0^{\circ} \mathrm{C}$} \\
\hline \multicolumn{2}{|c|}{ Adsorption } & \multicolumn{2}{|c|}{ Desorption } \\
\hline Mass [g] & Pressure [bar] & Mass [g] & Pressure [bar] \\
\hline 0 & 0 & 4.897062 & 1.900294 \\
\hline 0.346471 & 0.088002 & 4.417348 & 1.800782 \\
\hline 0.602398 & 0.18717 & 4.100943 & 1.785174 \\
\hline 0.957718 & 0.369203 & 3.876451 & 1.770907 \\
\hline 1.267057 & 0.497385 & 3.698664 & 1.767002 \\
\hline 2.388266 & 0.967485 & 3.592411 & 1.750488 \\
\hline 2.444496 & 1.043073 & 3.416483 & 1.711332 \\
\hline 2.532074 & 1.123585 & 3.25845 & 1.526379 \\
\hline 2.614094 & 1.16122 & 2.945375 & 1.162089 \\
\hline 2.732095 & 1.185121 & 2.79724 & 1.124889 \\
\hline 2.796942 & 1.223802 & 2.658612 & 1.105671 \\
\hline 2.844047 & 1.256887 & 2.523366 & 1.062386 \\
\hline 2.925639 & 1.271428 & 2.402748 & 0.958922 \\
\hline 3.083884 & 1.436101 & 2.306515 & 0.837199 \\
\hline 3.178109 & 1.604782 & 2.203548 & 0.740988 \\
\hline 3.307047 & 1.754341 & 2.129802 & 0.679066 \\
\hline 3.521779 & 1.831561 & 2.06286 & 0.646679 \\
\hline 3.878083 & 1.8687 & 1.927861 & 0.611433 \\
\hline 4.364449 & 1.893407 & 1.781937 & 0.595897 \\
\hline \multirow[t]{8}{*}{4.897062} & 1.900294 & 1.630939 & 0.584955 \\
\hline & & 1.460837 & 0.557341 \\
\hline & & 1.347789 & 0.526198 \\
\hline & & 1.253861 & 0.493781 \\
\hline & & 1.110644 & 0.450679 \\
\hline & & 0.905612 & 0.35223 \\
\hline & & 0.738138 & 0.286989 \\
\hline & & 0.598294 & 0.207759 \\
\hline
\end{tabular}

\begin{tabular}{|c|c|c|c|}
\hline \multicolumn{4}{|c|}{$20.0{ }^{\circ} \mathbf{C}$} \\
\hline \multicolumn{2}{|c|}{ Adsorption } & \multicolumn{2}{c|}{ Desorption } \\
\hline Mass [g] & Pressure [bar] & Mass [g] & Pressure [bar] \\
\hline 0 & 0 & 4.530322 & 2.263346 \\
\hline 0.339795 & 0.103749 & 4.394451 & 2.216079 \\
\hline 0.573314 & 0.20663 & 4.309986 & 2.145075 \\
\hline 0.834848 & 0.35865 & 3.713588 & 2.071357 \\
\hline 0.900618 & 0.385584 & 3.386066 & 1.946512 \\
\hline 1.069037 & 0.48982 & 3.199599 & 1.599301 \\
\hline
\end{tabular}




\begin{tabular}{|c|c|c|c|}
\hline 1.147164 & 0.534876 & 3.07535 & 1.41348 \\
\hline 1.241136 & 0.577956 & 2.928329 & 1.35167 \\
\hline 1.361809 & 0.616382 & 2.816385 & 1.335169 \\
\hline 1.42626 & 0.64603 & 2.681751 & 1.292356 \\
\hline 1.527298 & 0.653846 & 2.496915 & 1.21609 \\
\hline 1.568066 & 0.691083 & 2.35581 & 1.040209 \\
\hline 1.764852 & 0.717058 & 2.255617 & 0.902799 \\
\hline 1.918775 & 0.726213 & 2.107597 & 0.777476 \\
\hline 2.303926 & 0.96549 & 1.950938 & 0.736545 \\
\hline 2.314692 & 1.014553 & 1.875506 & 0.719199 \\
\hline 2.365973 & 1.088501 & 1.777669 & 0.709565 \\
\hline 2.404681 & 1.14625 & 1.613185 & 0.685171 \\
\hline 2.435903 & 1.20665 & 1.50836 & 0.66211 \\
\hline 2.470878 & 1.254777 & 1.351764 & 0.621723 \\
\hline 2.527806 & 1.332827 & 1.192736 & 0.554773 \\
\hline 2.643623 & 1.387614 & 1.185756 & 0.56281 \\
\hline 2.735131 & 1.420049 & 1.04395 & 0.475275 \\
\hline 2.89669 & 1.521702 & 0.860739 & 0.371313 \\
\hline 2.923232 & 1.560493 & 0.661015 & 0.256965 \\
\hline 2.97927 & 1.580197 & 0.022447 & 0.001243 \\
\hline 3.074567 & 1.698742 & & \\
\hline 3.166448 & 1.891045 & & \\
\hline 3.240358 & 2.031221 & & \\
\hline 3.562047 & 2.189398 & & \\
\hline 4.319476 & 2.269476 & & \\
\hline 4.530322 & 2.263346 & & \\
\hline
\end{tabular}

7. Data for the n-butane isotherms measured in the $\mathrm{S}+\mathrm{M}$ mixture

\begin{tabular}{|c|c|c|c|}
\hline \multicolumn{4}{|c|}{$9.1{ }^{\circ} \mathbf{C}$} \\
\hline \multicolumn{2}{|c|}{ Adsorption } & \multicolumn{2}{c|}{ Desorption } \\
\hline Mass [g] & Pressure [bar] & Mass [g] & Pressure [bar] \\
\hline 0 & 0 & 6.235701 & 1.547102 \\
\hline 0.208776 & 0.020295 & 6.080808 & 1.464849 \\
\hline 0.46184 & 0.069728 & 5.671322 & 1.426921 \\
\hline 0.708836 & 0.13105 & 5.153411 & 1.410231 \\
\hline 0.990444 & 0.21401 & 4.2458 & 1.406813 \\
\hline 1.068584 & 0.26335 & 3.997515 & 1.404092 \\
\hline 1.285961 & 0.302455 & 3.853871 & 1.396206 \\
\hline 1.5617 & 0.373035 & 3.726453 & 1.376158 \\
\hline 1.733702 & 0.397855 & 3.636176 & 1.35395 \\
\hline 1.922162 & 0.423755 & 3.534045 & 1.285517 \\
\hline
\end{tabular}




\begin{tabular}{|c|c|c|c|}
2.002139 & 0.426759 & 3.47595 & 1.216162 \\
\hline 2.248015 & 0.449339 & 3.419768 & 1.138278 \\
\hline 2.409273 & 0.450511 & 3.370835 & 1.035298 \\
\hline 2.505937 & 0.467859 & 3.350107 & 0.986016 \\
\hline 2.576581 & 0.472374 & 3.329936 & 0.946681 \\
\hline 2.823773 & 0.527626 & 3.286636 & 0.871126 \\
\hline 3.054789 & 0.657265 & 3.125771 & 0.667877 \\
\hline 3.212355 & 0.883471 & 3.062765 & 0.577443 \\
\hline 3.196419 & 0.970459 & 3.015315 & 0.535185 \\
\hline 3.248961 & 1.121785 & 2.764703 & 0.46388 \\
\hline 3.307963 & 1.252389 & 2.548045 & 0.456267 \\
\hline 3.355342 & 1.336384 & 2.432809 & 0.439411 \\
\hline 3.419438 & 1.406853 & 2.105425 & 0.413251 \\
\hline 3.823851 & 1.4891 & 1.872604 & 0.394286 \\
\hline 5.568206 & 1.512536 & 1.564201 & 0.356318 \\
\hline 6.10954 & 1.53025 & 1.374254 & 0.318619 \\
\hline 6.235701 & 1.547102 & 1.108685 & 0.236948 \\
\hline
\end{tabular}

\begin{tabular}{|c|c|c|c|}
\hline \multicolumn{4}{|c|}{$\mathbf{1 3 . 1}^{\circ} \mathbf{C}$} \\
\hline \multicolumn{2}{|c|}{ Adsorption } & \multicolumn{2}{c|}{ Desorption } \\
\hline Mass [g] & Pressure [bar] & Mass [g] & Pressure [bar] \\
\hline 0 & 0 & 5.797981 & 1.749849 \\
\hline 0.855191 & 0.210267 & 5.538468 & 1.672083 \\
\hline 0.88454 & 0.238698 & 5.229218 & 1.626965 \\
\hline 0.908942 & 0.241168 & 4.747438 & 1.622235 \\
\hline 1.37511 & 0.389628 & 4.424205 & 1.622481 \\
\hline 1.414239 & 0.417594 & 4.086416 & 1.608738 \\
\hline 1.601043 & 0.455882 & 3.905499 & 1.598109 \\
\hline 1.860075 & 0.487893 & 3.681765 & 1.571664 \\
\hline 2.165463 & 0.531443 & 3.63732 & 1.551134 \\
\hline 2.498441 & 0.564507 & 3.579074 & 1.509384 \\
\hline 2.871923 & 0.62669 & 3.524761 & 1.424565 \\
\hline 3.018418 & 0.794436 & 3.466163 & 1.349452 \\
\hline 3.120778 & 0.894065 & 3.416893 & 1.172962 \\
\hline 3.201362 & 1.094083 & 3.408822 & 1.195236 \\
\hline 3.22673 & 1.193803 & 3.373914 & 1.126904 \\
\hline 3.249652 & 1.27361 & 3.3235 & 1.005106 \\
\hline 3.324971 & 1.433406 & 3.283706 & 0.961226 \\
\hline 3.368802 & 1.512507 & 3.206202 & 0.930699 \\
\hline 3.766387 & 1.666537 & 3.190979 & 0.933098 \\
\hline
\end{tabular}




\begin{tabular}{|l|c|c|c|}
4.621998 & 1.687816 & 3.1207 & 0.749098 \\
\hline 5.561741 & 1.72642 & 3.072847 & 0.659922 \\
\hline 5.797981 & 1.749849 & 2.96948 & 0.608347 \\
\hline & & 2.734571 & 0.551467 \\
\hline & & 2.585643 & 0.542018 \\
\hline & & 2.315457 & 0.516196 \\
\hline & & 1.956688 & 0.476358 \\
\hline & & 1.704906 & 0.452104 \\
\hline & & 1.456787 & 0.396076 \\
\hline & & 1.237654 & 0.315416 \\
\hline & & 0.991221 & 0.232013 \\
\hline & & 0.68714 & 0.136428 \\
\hline
\end{tabular}

\begin{tabular}{|c|c|c|c|}
\hline \multicolumn{4}{|c|}{16.0} \\
\hline \multicolumn{2}{|c}{ Adsorption } & \multicolumn{2}{c|}{ Desorption } \\
\hline Mass [g] & Pressure [bar] & Mass [g] & Pressure [bar] \\
\hline 0 & 0 & 5.720352 & 1.918302 \\
\hline 0.405132 & 0.093402 & 5.320781 & 1.80104 \\
\hline 0.718451 & 0.190663 & 4.815202 & 1.785342 \\
\hline 1.151789 & 0.371732 & 4.339611 & 1.775411 \\
\hline 1.566318 & 0.505034 & 3.946362 & 1.762548 \\
\hline 3.074371 & 0.968212 & 3.77462 & 1.749511 \\
\hline 3.107615 & 1.039975 & 3.605993 & 1.710851 \\
\hline 3.159447 & 1.191334 & 3.459208 & 1.526121 \\
\hline 3.213128 & 1.327443 & 3.274498 & 1.160253 \\
\hline 3.25139 & 1.439814 & 3.245082 & 1.131889 \\
\hline 3.296537 & 1.573166 & 3.199655 & 1.103294 \\
\hline 3.359257 & 1.685892 & 3.154795 & 1.064814 \\
\hline 3.456335 & 1.793003 & 3.102118 & 0.960239 \\
\hline 3.621056 & 1.85353 & 3.044954 & 0.843847 \\
\hline 4.733543 & 1.904957 & 2.981987 & 0.73757 \\
\hline 5.097775 & 1.909767 & 2.90305 & 0.682952 \\
\hline 5.720352 & 1.918302 & 2.830128 & 0.646934 \\
\hline & & 2.686633 & 0.612392 \\
\hline & & 2.538728 & 0.607292 \\
\hline & & 2.352687 & 0.591788 \\
\hline & & 2.091654 & 0.559715 \\
\hline & & 1.839727 & 0.522752 \\
\hline & & 1.647528 & 0.497429 \\
\hline & & 1.423974 & 0.450344 \\
\hline
\end{tabular}




\begin{tabular}{|l|l|l|l|} 
& & 1.148743 & 0.349748 \\
\hline & & 0.956151 & 0.285314 \\
\hline & & 0.774925 & 0.205689 \\
\hline
\end{tabular}

\begin{tabular}{|c|c|c|c|}
\hline \multicolumn{5}{|c|}{$\mathbf{1 6 . 0}{ }^{\circ}$ C } \\
\hline \multicolumn{2}{|c|}{ Adsorption } & \multicolumn{2}{c|}{ Desorption } \\
\hline Mass [g] & Pressure [bar] & Mass [g] & Pressure [bar] \\
\hline 0 & 0 & 3.771836 & 2.248281 \\
\hline 0.388845 & 0.100728 & 3.763157 & 2.262614 \\
\hline 0.693795 & 0.20689 & 3.708913 & 2.211213 \\
\hline 1.023687 & 0.355927 & 3.631126 & 2.138297 \\
\hline 1.071414 & 0.38157 & 3.523518 & 2.044083 \\
\hline 1.28138 & 0.480573 & 3.46167 & 2.062609 \\
\hline 1.424381 & 0.529616 & 3.400206 & 1.939984 \\
\hline 1.511687 & 0.571197 & 3.298815 & 1.59193 \\
\hline 1.750508 & 0.605708 & 3.224897 & 1.406749 \\
\hline 1.815978 & 0.638101 & 3.18069 & 1.340324 \\
\hline 1.921223 & 0.633784 & 3.164453 & 1.326751 \\
\hline 2.0452 & 0.659109 & 3.120083 & 1.284652 \\
\hline 2.059877 & 0.686656 & 3.082444 & 1.214491 \\
\hline 2.350073 & 0.711938 & 3.015954 & 1.037683 \\
\hline 2.509574 & 0.727644 & 2.94162 & 0.89142 \\
\hline 3.034414 & 0.958476 & 2.813349 & 0.764604 \\
\hline 3.063246 & 1.033653 & 2.658312 & 0.727221 \\
\hline 3.102467 & 1.129386 & 2.564516 & 0.723777 \\
\hline 3.148966 & 1.251328 & 2.4688 & 0.708798 \\
\hline 3.208866 & 1.423782 & 2.269969 & 0.685233 \\
\hline 3.235666 & 1.511016 & 2.092997 & 0.661542 \\
\hline 3.277562 & 1.629351 & 1.772289 & 0.612004 \\
\hline 3.312782 & 1.75746 & 1.466756 & 0.541749 \\
\hline 3.372997 & 1.914327 & 1.468613 & 0.557647 \\
\hline 3.449509 & 2.032478 & 1.260092 & 0.474944 \\
\hline 3.535427 & 2.096312 & 1.03531 & 0.364253 \\
\hline 3.539504 & 2.138234 & 0.781093 & 0.248526 \\
\hline 3.76206 & 2.298635 & & \\
\hline 3.771836 & 2.248281 & & \\
\hline & & & \\
\hline & & & \\
\hline & & & \\
\hline & & & \\
\hline & & & \\
\hline
\end{tabular}

